Rhode Island College

Digital Commons @ RIC

$5-14-2017$

\title{
Evaluation of a Diabetes Management Program for Persons with Serious and Persistent Mental IIIness
}

Peris W. Mwangi

Rhode Island College

Follow this and additional works at: https://digitalcommons.ric.edu/etd

Part of the Mental and Social Health Commons, and the Nursing Commons

\section{Recommended Citation}

Mwangi, Peris W., "Evaluation of a Diabetes Management Program for Persons with Serious and Persistent Mental Illness" (2017). Master's Theses, Dissertations, Graduate Research and Major Papers Overview. 195.

https://digitalcommons.ric.edu/etd/195

This Major Paper is brought to you for free and open access by the Master's Theses, Dissertations, Graduate Research and Major Papers at Digital Commons @ RIC. It has been accepted for inclusion in Master's Theses, Dissertations, Graduate Research and Major Papers Overview by an authorized administrator of Digital Commons @ RIC. For more information, please contact digitalcommons@ric.edu. 


\section{EVALUATION OF A DIABETES MANAGEMENT \\ PROGRAM FOR PERSONS WITH SERIOUS \\ AND PERSISTENT MENTAL ILLNESS}

by

Peris W. Mwangi

A Major Paper Submitted in Partial Fulfillment

of the Requirements for the Degree of

Master of Science in Nursing

in

The School of Nursing

Rhode Island College

2017 


\begin{abstract}
Clinical Practice Recommendations and Evidence-Based Guidelines for Integrated Care were used to evaluate a diabetes management program for persons with serious and persistent mental illness in an integrated primary and behavioral health care center in New England. A simple random method was used to select a sample of 25 medical records of patients with diabetes and at least one mental illness. Data on seven diabetes content areas offered to patients and biophysical measures of weight, body mass index (BMI) and Hemoglobin A1C were collected via retrospective electronic chart reviews. Results showed the center focused on nutrition and exercise education, offered to $90 \%$ and $85 \%$ of patients respectively. Other education areas, including medications, selfmonitoring of blood sugar, foot care, dental care and smoking cessation were offered to $5 \%-40 \%$ of the patients. An unexpected finding was noted, in that most participants (65\%) gained weight despite focused nutrition and exercise education. The BMI was consequently elevated at a median level of 35 , identified as obese. In spite of the weight and BMI increase, $65 \%$ of the patients had well controlled diabetes with an A1C below 7.The finding may be attributed to compliance and personalized diabetes medication regimen. Keeping all appointments did not improve biophysical measures: $67 \%$ of those who kept all appointments gained weight and increased A1C from base line. The unexpected results underscore the complexity and confounding nature of factors influencing diabetes in this population despite measures to improve health outcomes.
\end{abstract}




\section{Acknowledgements}

I would like to thank Dr. Padula and Dr. Schwager for their extraordinary support in completion of this project. Thanks to Dr. Mock, Deb Burbank and Dr. Jim Howard for their expert advice. Thanks to friends and family who stepped in to help with household responsibilities during this tasking period. I would like to thank my dear husband Jacob and children Lisa, Elsie and Josh who sacrificed a lot and cheered me along the way.

Finally I am very thankful for God's amazing grace and strength that has carried me thus far. 


\section{Table of Contents}

Background/Statement of the Problem .................................. 1

Literature Review....................................................

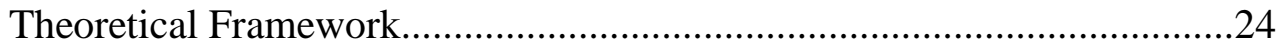

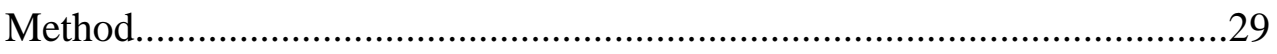

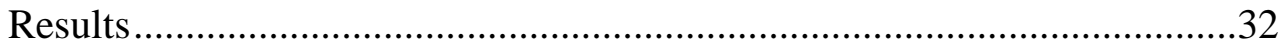

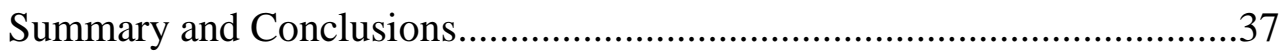

Recommendations and Implications for Advanced Nursing Practice..........42

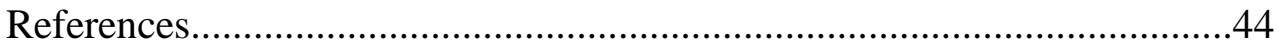

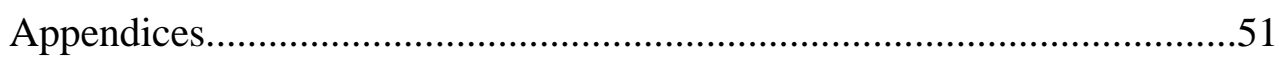


Evaluation of a Diabetes Management Program for Persons with

Serious and Persistent Mental Illness

\section{Background/Statement of the Problem}

Chronic diseases are the leading causes of illness, disability, and death in North America (Agency for Healthcare Research and Quality [AHRQ], 2013). One of the strategies identified to improve the health of people with comorbidities is case management, also known as integrated care coordination. The AHRQ (2013) defined case management as a "strategy for improving patient care through designating a member of the health care team to manage multiple aspects of a patient's care, including planning and assessment, coordination of services, patient education, and clinical monitoring"

(p.1). Case management has been demonstrated to result in improved patient outcomes at reduced cost. The Intermountain Healthcare conducted a retrospective, longitudinal, cohort study to assess the association of integrated team-based care with patient outcomes and costs. Results showed improved clinical outcomes and financial benefits (ReissBrennan et al., 2016).

Diabetes is prevalent among people with psychotic disorders such as schizophrenia and schizoaffective disorder, as well as adults with affective disorders including bipolar, depression and anxiety disorders (Ward \& Druss, 2015). It is, however, difficult to estimate the prevalence with precision as diabetes is under-diagnosed in patients with psychotic disorders (Ward \& Druss). Individuals with serious and persistent mental illness (SPMI) are less likely to be offered screenings than the general population routinely receives including cholesterol, weight and urine checks or even advice on 
smoking cessation (Hemingway, Trotter, Stephenson, \& Holdich, 2013). A survey completed by Rethink Mental Illness (2009) found that only 52\% of people with SPMI were offered a physical examination in the last two years, suggesting this population is marginalized from mainstream preventive and screening services.

There is a complex, multifactorial association between diabetes and psychiatric disorders (Balhara, 2011). Comorbidity of diabetes and psychiatric disorders may exist as an independent condition with no apparent connection. In other cases, diabetes may contribute to the development of psychiatric disorders: various biological and psychological factors facilitate the emergence of a mental disorder (Balhara). Disorders like schizophrenia and depression are significant diabetes risk factors, as medications used to treat psychiatric disorders may result in impaired glucose tolerance. Abuse of alcohol and tobacco, common with psychiatric disorders, can alter the pharmacokinetics of oral diabetic agents. In addition, conditions like depression can influence treatment adherence, and certain phobias like fear of needles and injections can interfere with glucose monitoring and insulin administration (Balhara).

Extensive literature suggests individuals with SPMI are 2-3 times more likely than the general population to develop type 2 diabetes (De Hert et al., 2011). Risk factors seem to have synergetic effects and include: use of antipsychotic medications; genetics and adverse determinants of health, such as poor housing or homelessness; poor access and utilization of health services; and poverty (Ward \& Druss, 2015).

The influence of diabetes and psychiatric disorder comorbidities on public health and the economy is huge. This is evidenced by high cost of care which is at least twice that of the general population, impaired quality of life, poor treatment adherence and poor 
glycemic control leading to diabetes complications, frequent emergency room visits and hospitalizations (Centorrino, Mark, Talamo, Oh, \& Chang, 2009). The mortality rate for people with SPMI is 2-3 times higher than the general population, translating to 13-30 years shortened life expectancy (Colton \& Manderscheid, 2006). Recent studies suggest this gap may be increasing (Olfson, Gerhard, Huang, Crystal, Stroup, 2015). In their study entitled Premature mortality among adults with schizophrenia in the United States, Olfson et al. found adults with schizophrenia were more than 3.5 times as likely to die during the follow-up period as were adults in the general population. More than $85 \%$ of known causes of death were attributed to cardiovascular and respiratory diseases, different types of cancers and diabetes.

Promotion of healthy life style choices is paramount. There is evidence that providing guidance and advice on both physical and mental wellbeing to people with SPMI improves self-esteem and ability to make better choices (Hemingway et al., 2013). In addition, physical and emotional assessment for effects of psychotropic medications is essential for proper management of comorbidities and for averting complications (Hemingway et al.).

Diabetes is a condition that demands a total change in lifestyle, as daily management of the disease is required. Most individuals with SPMI have difficulties managing their basic daily lives due to cognitive deficits such as memory problems, sequencing, and executive functions. These are all essential for effective diabetes selfmanagement. Many of these people do not function independently and require the support of family, house managers, mental health workers and case managers (McDevitt, 
Snyder, Breitmayer, Paun, \& Wojciechowski, 2003). These multifactorial challenges make it extremely difficult to manage diabetes in this population.

In 2003, a multidisciplinary team at the University of Illinois, Chicago was formed to develop evidence-based practice guidelines for this vulnerable population. Advanced practice nurses at the Nursing Institute, College of Nursing and other healthcare practitioners led the initiative. They drew on their primary care and mental health expertise to develop Clinical Practice Recommendations and Evidence-Based Guidelines for Integrated Care (McDevitt et al., 2003). The goal of these guidelines was to provide expert advice for the management of type 2 diabetes in patients with serious and persistent mental illness.

The purpose of this project was to evaluate a diabetes management program for persons with serious and persistent mental illness, in an integrated primary and behavioral health care center in New England, using the Clinical Practice Recommendations and Evidence-Based Guidelines for Integrated Care developed by McDevitt et al. (2003).

Next, the review of the literature will be presented. 


\section{Literature Review}

A literature search for interventions and standards of care for people with diabetes and psychiatric disorders was conducted using the CINAHL, MEDLINE, and PubMed databases, including the period from 1990 to 2016. Key words used were mental illness, psychiatric disorders, serious mental illness, diabetes, chronic diseases, intervention, evaluation, standards, guidelines, treatment, self-management, care coordinators, and integrated care.

\section{Psychiatric Disorders in North America}

According to the Centers for Disease Control and Prevention (CDC, 2011), nearly $50 \%$ of adults in America will develop at least one mental disorder in their lifetime. The 2015 National Survey on Drugs and Health (2015) projected 43.4 million adults (17.9\%) had a mental illness, of which 9.8 million (4\%) were serious and persistent mental illness (Substance Abuse and Mental Health Services Administration [SAMHSA], 2015). The percentages of adults with any mental illness and those with SPMI remained stable from 2008 to 2015 . In 2015, 16.1 million adults $(6.7 \%)$ had at least one major depressive episode the previous year, and 10.3 million adults (4.3\%) had a major depressive episode with severe impairment in 2014. These percentages have been stable since 2005 (SAMHSA).

Substance abuse is prevalent among people with mental illness (SAMHSA, 2015). In $2014,3.3 \%$ (8.1 million adults) were estimated to have co-occurring mental and substance use disorder, 2.3 million (1\% of the adults) had SPMI and substance use disorder. Of these adults, only $48 \%$ and $62.6 \%$ respectively received treatment at a mental health or specialty facility (SAMHSA). The cost of mental health and substance 
use disorder treatment is expected to total $\$ 280.5$ billion in 2020 , an increase from $\$ 171.7$ billion in 2009 (SAMHSA, 2014). The treatment expenditure growth curve is expected to slow down from recent trends and lag behind all/general health spending curve to average an annual growth of $4.6 \%$. All-health spending growth rate of $5.8 \%$ is expected from 2009-2020 (SAMHSA). The changes are attributed to the Affordable Care Act, closure of state psychiatric hospital beds (nine state hospitals and $9 \%$ of state hospital beds closed between 2009 and 2012) and the loss of patent protection for large number of prescription drugs used to treat mental illness and substance abuse disorders. The patent loss allowed entry of lower cost generic drugs into the market (SAMHSA).

\section{Psychological Impact of SPMI}

Mental illnesses often affect motivation, which hinders self-confidence and consequently the ability to care for one's self. (McDevitt et al., 2003). Schizophrenia is particularly difficult to treat and is the most impairing of psychiatric conditions.

Symptoms include deficient speech, lack of interest/pleasure, flat affect, inability to initiate or participate in activities, disturbed perception and ideation, impaired memory and bizarre behavior (McDevitt et al.). Lack of motivation is attributed to poor selfefficacy.

According to Bandura (1977), self-efficacy refers to one's belief or perception of their ability to succeed in specific situations or accomplish particular tasks. The determination and effort spent on a task is influenced by one's confidence in their ability to succeed. When an individual perceives low chances of succeeding, avoidance behavior is exhibited. Self-efficacy influences coping with chronic conditions (Bandura). When people with SPMI perceive they have social support and the ability to use problem- 
focused coping strategies, they adopt better to daily stress (Macdonald, Pica, McDonald, Hayes, \& Baglioni, 1998). People with SPMI struggle with high levels of anxiety, selfcontrol and incorrect self-perception. These in turn affect their ability to engage in normal functions (McDevitt et al., 2003).

Cognitive functions in people with SPMI vary widely depending on the cognitive deficits that are characteristic of the different mental conditions (McDevitt et al., 2003). The deficits influence the level of learning, ability to implement a plan and consequently affect the outcomes. The presence and extent of cognitive deficits should be assessed and considered when planning interventions and follow up. It is important to assess, monitor and control cognitive symptoms, as they affect a person's functional level. Persons with schizophrenia and schizoaffective disorder (combination of schizophrenia and mood disorder symptoms) perform poorly with all tasks, but atypical antipsychotic drugs can be used to improve their cognitive functioning (McDevitt et al.).

Many people with SPMI are unable to accept their diagnosis and, therefore, are noncompliant with treatment (Amador et al., 1994). Insight and awareness allow the patient to understand the nature and consequences of their disorder, as well as identify the symptoms of their condition. Insight refers to the patient's agreement with the provider's view of diagnosis and proposed treatment, while awareness refers to acknowledgement of the illness and its contribution to their current situation. Patients with poor insight and awareness, as seen in schizophrenia, have poor motivation and psychosocial functioning (Amador et al.).

Emotions also affect an individual's ability to solve problems (Huppert, Weiss, Lim, Pratt, \& Smith, 2001). Feelings may be aroused by the problem itself, appraisal of 
the problem, one's perception of the need to solve it and the approach used to address the problem. Emotions prior to and during a problem will inhibit or facilitate successful problem solving processes. In addition, depression, hopelessness, helplessness and anxiety inhibit problem solving ability; and individuals may perceive loss of control and give up. Depression and anxiety are also associated with lower satisfaction and poor quality of life (Huppert et al.).

\section{Guidelines and Standards}

The International Diabetes Federation (IDF) Task Force developed evidencebased Global Guidelines for the care of people with type 2 diabetes. The recommendations were established on three levels: standard, comprehensive and minimal (IDF, 2006). The Global Guidelines address 19 topics under the three levels of care. They include different diabetes populations, screening and diagnosis, different modes of treatment, self-management, lifestyle management and education, complications of diabetes, and monitoring diabetes in different settings.

Standard care is cost-effective evidence-based care, utilized in nations with a well-developed service base and health care funding system. Minimal care is provided in health care settings with very limited resources. It is the lowest level of care that anyone with diabetes should receive. Comprehensive care provides the latest and complete range of health technologies to people with diabetes, with the aim of achieving the best possible care outcomes. The evidence-base supporting the use of these expensive or new technologies, however, is relatively weak (IDF, 2006).

The National Standards for Diabetes Self-Management Education and Support (Funnell et al., 2010) identify diabetes self-management education (DSME) as a critical 
element in the care of people with diabetes and those at risk for developing the disease. Implementing DSME helps prevent or delay the complications of diabetes. The national standards are designed to define quality DSME and support diabetes educators in providing evidence-based education and self-management support (Funnell et al.). Diabetes self-management education (DSME) refers to activities that assist persons with prediabetes or diabetes to implement and sustain behaviors needed to manage diabetes on an ongoing basis. Behavioral, educational, psychosocial, or clinical support is offered (Haas et al., 2012). This evidence-based education develops the knowledge, skill, and ability necessary for diabetes self-care; while incorporating the needs, goals, and life experiences of the person with diabetes (Funnell et al., 2010). The objectives of DSME are to support informed decision-making, self-care behaviors, problem-solving and active collaboration with the health care team; thereby improving clinical outcomes, health status, and quality of life (Funnell et al.).

Ten standards of DSME are stipulated in the National Guidelines (Haas et al., 2012).

(1). Addresses the internal structure of the organization, mission statement and goals that lead to effective and efficient provision of DSME and diabetes selfmanagement support (DSMS).

(2). The external input standard promotes program quality through stakeholder and expert engagement.

(3). Access standard focuses on the target population, teaching approaches/ strategies and supports resources available. 
(4). The program coordination standard is responsible for planning, implementation and evaluation of educational services.

(5). Recommends that instructional staff be certified in diabetes care and education.

(6). A written curriculum reflecting current evidence, practice guidelines and criteria for evaluating outcomes should be utilized.

(7). Focuses on individualized education and a support plan aimed at behavior change.

(8). The participant's ongoing support standard focuses on outcomes goals and plans for ongoing self-management support.

(9).Recommends monitoring the participant's progress and evaluating the effectiveness of the educational intervention.

(10). The last standard addresses quality improvement, and seeks to identify and improve gaps in services or quality using a systematic review of process and outcome data (Haas et al.).

The American Diabetes Association (ADA) Standards of Medical Care in Diabetes (ADA, 2016), formally known as Clinical Practice Recommendations, are reviewed and published annually. The standards include the most current evidence based recommendations for diagnosing and treating type 1 and type 2 diabetes in all populations. The recommendations are very comprehensive and cover most areas of concerns for a person living with diabetes. The standards start with improving care for a person with prediabetes, then moves on to recommendations for diagnosis and treatment 
strategies, hospitalization, transition to rehabilitation, and advocacy. The

recommendations follow the disease process and continuum of care model (ADA, 2016).

\section{Psychological Aspects of Diabetes Management}

An important goal of diabetes education and care is to enhance individuals' selfefficacy to manage their condition. This in turn drives their will and ability to make daily decisions regarding glucose monitoring, nutrition, medications, physical activity, stress management, and interaction with their healthcare providers and support systems (Anderson, Funnell, Fitzgerald, \& Marrero, 2000). In a study of psychosocial selfefficacy, Anderson et al. assessed 375 patients on managing the psychosocial aspects of diabetes, dissatisfaction and readiness to change, and setting and achieving diabetes goals. Psychosocial self-efficacy refers to one's ability to successfully address psychosocial issues like managing stress, dealing with uncomfortable situations, and obtaining support (Anderson et al.).

The study utilized Diabetes Empowerment Scale (DES), a measure of diabetesrelated psychosocial self-efficacy, with 37 item Likert-type questionnaire based on three DES subscales (Anderson et al., 2000). Managing the Psychosocial Aspects of Diabetes subscale assesses the perceived ability to obtain social support, manage stress, selfmotivate, and make right diabetes-related decisions. The second subscale, Assessing Dissatisfaction and Readiness to Change, assesses perceived ability to identify aspects of managing diabetes that patients are dissatisfied with and their ability to determine when they are ready to change their diabetes self-management plan. The third subscale, Setting and Achieving Diabetes Goals, assesses patients' perceived ability to set realistic goals and reach them by overcoming barriers to achieving their goal (Anderson et al.). 
Questionnaires were mailed or given to a convenience sample population involved in Michigan Diabetes Research and Training Center Outreach Programs. The findings were analyzed and the three DES subscales correlated with validating subscales from Diabetes Care Profile (DCP), namely Positive Attitude, Negative Attitude, and Diabetes Understanding subscales. All correlations were significant at $\mathrm{p}<0.0001$. The correlations (0.32 -0.59) with the Positive Attitude scale indicated that the patients reporting greater levels of psychosocial self-efficacy had a more positive outlook about their life and diabetes. The correlations with the Negative Attitude Scale (0.38-0.59) showed patients who reported lower levels of psychosocial self-efficacy had a negative outlook on their life and diabetes. There was a positive correlation (0.39-0.43) with the self-reported Diabetes Understanding Scale had a small positive correlation (0.10-0.17) with level of education. Patients reporting greater levels of psychosocial self-efficacy also reported having a better understanding of diabetes (Anderson et al.).

Positive psychological health is crucial in sustaining continued long-term coping efforts and protecting patients from the adverse impact of prolonged emotional disorder and illness perception (Chew, Shariff-Ghazali, Fernandez, 2014). An international survey, the Diabetes Attitudes, Wishes and Needs second study (DAWN2), was conducted to assess the psychosocial outcomes in 8596 adults with diabetes across 17 countries in four continents. (Nicolucci et al., 2013). Questionnaires assessing healthrelated quality of life, social support, self-management, priorities for improving diabetes care and attitudes/beliefs were conducted online, by telephone or in person. The results showed $13.8 \%(\mathrm{n}=1285)$ of the participants were depressed, $44.6 \%(\mathrm{n}=3486)$ reported diabetes-related distress, and $12.2 \%(\mathrm{n}=1193)$ rated their quality of life as poor or very 
poor. About $40 \%(n=2589)$ reported medication interfered with their ability to live a normal life. The availability of patient-centered chronic illness care and support for active involvement was rated as low. Most participants followed self-care advice for medication and diet and few reported adhering to glucose monitoring and foot examination advice. Only $48.8 \%(\mathrm{n}=5155)$ of respondents participated in diabetes educational programs/activities to help manage their diabetes. Negative impact was reported on all aspects investigated, ranging from $20.5 \%$ on relationship with family and friends to $62.2 \%$ on physical health (Nicolucci et al.).

Patients who use negative coping strategies perceive diabetes will negatively impact their future and are not motivated to manage the disease (Walker et al., 2012). Untreated psychosocial disorders in patients with diabetes may lead to more physical symptoms and complications.

\section{Health Education for Persons with SPMI and Diabetes}

Provision of appropriate health education is essential for diabetes selfmanagement. According to the National Standards for Diabetes Self-management Education (Funnell et al., 2010), diabetes education improves clinical outcomes and quality of life. Different educational programs and approaches that incorporate behavioral and psychosocial strategies exhibit improved clinical outcomes. The standards require that educational methods and materials used be suitable for the patient's age, culture, and learning needs (Funnell et al., 2010; McDevitt et al., 2003).

Targeted Training in Illness Management (TTIM) is an innovative technique that combines cognitive and social approaches to treat individuals with serious mental illness and diabetes, with a goal of improving mental and general health outcomes. (Sajatovic et 
al., 2011). An uncontrolled pilot trial of TTIM was carried out in a primary care setting with a sample of 12 patients with serious mental illness and diabetes. The TTIM project was carried out in two phases: 12 weekly group sessions and four weekly telephone follow-up sessions. Each group session was 60-90 minutes long, had six participants and was led by a nurse and peer educator. The education content included food and nutrition, stress and diabetes management. Baseline information on functional and general health status; impact of impairment; hemoglobin A1C (HbA1c), body mass index and health behaviors was obtained and reassessed at 12 and16 weeks.

At baseline, nine out of 12 participants were obese with a mean BMI 36; half of the participants had poorly controlled diabetes $(\mathrm{HbA} 1 \mathrm{C}>8)$, and five had baseline high blood pressure. On average, they had moderate degrees of psychopathology, defined as mental distress and maladaptive behavior, as documented by the Brief Psychiatric Rating Scale (BPRS) and the Montgomery-Asberg Depression (MADRS) Rating Scale. The scores were 37 and 23.5 respectively at baseline. Possible MADRS scores range from 0 to 60 , with higher scores indicating greater severity of depression. Scores for BPRS range from 18 to 126, with higher scores representing greater mental illness severity. Selfreported mental health status (SF-12 mental component summary [MCS]) 32.8 was substantially below the general US population by almost two standard deviations. Score for physical health status (SF-12 physical component summary [PCS]) 32.4 was also below the average level of the general population at baseline. Possible scores on mental and physical components range from 0 to 100 , with higher scores indicating higher selfreported mental and physical health status. 
Results of the intervention showed relevant improvements on most measures. There was no significant weight loss by the week 16, probably because TTIM did not focus on weight. Hemoglobin A1C for eight participants improved significantly by $67 \%$, three participants increased the A1C slightly and one participant had no change (had normal A1C). There was a 15\% mean reduction in BPRS (31.50) and $48 \%$ mean reduction in MADRS (12.08) scores meaning decreased mental distress and abnormal behaviors. Self-reported mental health status (34.61) improved by $7 \%$ and by $15 \%$ in self-reported physical health scores (37.84) (Sajatovic et al.).

Multidimensional approaches that simultaneously target mental health and general medical health such as TTIM motivates individuals to take active roles in their care can be effective (Sajatovic et al., 2011). Social skills training for people with severe mental illness promote social competence which allows successful daily living (Kopelowicz, Liberman, Zarate, 2006). Individualized coaching and reinforcement are necessary to apply and maintain learning. Simple tasks with a high likelihood of success are introduced first and then higher task demands are gradually introduced to maintain success. This is only possible where an ongoing patient-provider relationship exists (Bachrach, 2000; McDevitt et al., 2003).

\section{Integrated Care for Persons with SPMI and Diabetes}

Management of diabetes in the context of SPMI represents arduous challenges. The features and consequences of SPMI impair general daily self-care and certainly affect diabetes self-management. Due to impaired cognitive function, individuals may experience difficulties making independent decisions, setting goals, planning and problem solving; resulting in poor self-care skills (McDevitt et al., 2003). Since diabetes 
management is a part of everyday living, it is crucial that individuals maintain effective self-care. Primary providers should, therefore, closely monitor each patient's mental status and its effect on self-care. Integrated physical and mental care, where the primary and mental providers, caseworker, and patient work directly together has been shown to have better overall self-care outcomes (McDevitt et al.).

A 10-year (2003-2013) retrospective, longitudinal, study conducted by Intermountain Healthcare showed clinical and financial benefits of an integrated delivery system (Reiss-Brennan et al., 2016). The study measured 113,452 adults receiving care from 113 primary care practices at Intermountain Healthcare: 27 integrated team-based care (TBC) practices and 75 traditional practice management (TPM) practices (usual care). The TBC practices integrated physical and mental health and provided the dual care routinely. The primary care provider, mental health provider case manager and the patient worked together to manage health conditions and engage patients in their care. Traditional practice management treated patients in internal medicine, geriatric practices and family practices. Patients were assigned to TPM or TBC annually based on the primary care practice visited.

A retrospective chart review was conducted for the period 2003- 2013. Information collected included: screening and treatment for depression and diabetes; comorbidities; self-reported adherence to diabetes care protocols including regular blood sugar testing; and use of diabetes management care plans. Data on all hospital admission, emergency department visits and ambulatory visits were obtained. Insurance payment data were collected to assess actual re imbursements received. Baseline demographics 
and clinical characteristics showed that patients in the TBC group had more chronic diseases, including diabetes, depression, high blood pressure and other comorbidities. Results showed the integrated team based care had better performance outcomes than the traditional practice. Team-based care screened and diagnosed $46.1 \%$ of the patients with active depression compared to $24.1 \%$ in traditional practices. This allowed the provider to offer early medical and behavioral interventions. Team-based patients' adherence to diabetes care protocols, including regular blood sugar testing was $24.6 \%$ compared to $19.5 \%$ in traditional practices. This was attributed to patients' engagement with care teams to manage their health. Only $8.7 \%$ of patients in traditional practices had a documented self-care plan to help manage their health conditions, compared to $48.4 \%$ patients in team-based practices (Reiss-Brennan et al., 2016).

High blood pressure was used as a control variable, meaning the team-based care model was not utilized for blood pressure management. Findings showed $85 \%$ of patients in the TBC had controlled high blood pressure, compared to $97.7 \%$ in traditional practices. As predicted, elevated blood pressure management showed less improvement than conditions targeted by TBC such as diabetes and depression. Patients in TBC also used fewer health care services and had lower total costs, as shown by data per 100 person years. The rate of emergency room visits with TBC was 18.1 compared to 23.5 for patients in traditional practices, a 23\% reduction. The rate of hospital admissions was 9.5 for patients in TBC as opposed to 10.6 in TPM, which was a $10.6 \%$ reduction. Primary care physician encounters were 232.8 for patients in team-based practices compared to 250.4 for patients in traditional practices, a reduction of $7.0 \%$ acute care utilization. Payments to providers were $\$ 3,400$ for patients in team-based practices versus $\$ 3,515$ for 
patients in traditional practices, a savings of $3.3 \%$. These payments were less than the investment costs incurred by Intermountain Healthcare to create the team-based practice model (Reiss-Brennan et al., 2016).

\section{Clinical Practice Recommendations and Evidence-Based Guidelines for Integrated Care}

A multidisciplinary team at the University of Illinois developed Clinical Practice Recommendations and Evidence-Based Guidelines for Integrated Care for persons with diabetes and serious and persistent mental illness (McDevitt, et al., 2003). Nine recommendations were developed around four areas of intervention identified in the patient need assessment. The areas were affective support, health information, decisional control and professional-technical competency.

Affective support. The first recommendation is the provision of integrated care where the primary and mental health providers and case managers work directly together under the same roof to provide care to patients with SPMI and improve clinical outcomes (McDevitt et al., 2003). This model of care ensures the patients receive all their care at one place reducing opportunities for missed appointments. People with SPMI often delay seeking health care, due to cognitive, behavioral, and social factors. The Intermountain Healthcare study (Reiss-Brennan et al., 2016) discussed above showed integrated care had significantly higher rates of quality care measures such diagnosis and treatment of diabetes and depression, diabetes management, reductions in acute care utilization and decreased cost compared to traditional care model.

Building therapeutic alliance is the second recommendation, achieved by assigning clients to one primary care provider in order to build trust and ensure continuity 
of care. (McDevitt et al., 2003). The health care provider should stablish and maintain a supportive, therapeutic association with the patient as this becomes the foundation of developing trust and conducting treatment (Bachrach, 2000). Patient should feel free to discuss feelings or negative experiences with treatment plans which may facilitate adherence to the treatment plan.

A study by Sylvia et al. (2013) examined the association of patients' perceptions of therapeutic alliance with their psychiatrist, medication adherence and care satisfaction over a period of one year. Data were examined from the Multicenter Systematic Treatment Enhancement Program for Bipolar Disorder, an effectiveness study investigating the course and treatment of bipolar disorder. A target population of 2371 received two questionnaires: the Care Satisfaction Questionnaire (CSQ) and the Helping Alliance Questionnaire (HAQ). The Care Satisfaction questionnaire, a 44 items self-rated scale assessed patients' perceptions of quality of care received, the degree to which they felt treated with "respect" and "courtesy," their behavioral health services and accessibility of psychiatrists. The Helping Alliance Questionnaire, a 19-item self-report questionnaire, captured key aspects of the therapeutic alliance namely patient's perception of psychiatrist and patients, nature of the patient-psychiatrist relationship, and patients' motivation for treatment.

Findings showed the patients' perceptions of collaboration, accessibility and empathy were significantly associated with adherence to treatment (Sylvia et al.). Perceptions of strong therapeutic relationship with the psychiatrist such as having a good relationship, feeling understood and having meaningful collaborations and exchanges were associated with medication and treatment adherence as evidenced by a significant 
OR $<1.0$ and $95 \%$ confidence interval. If patients felt respected and helped by their psychiatrists they were more likely to be adherent to treatment regardless of factors associated with poor adherence such as alcohol use disorder, earlier onset of illness, current anxiety, or current mood. Patient perception of the psychiatrist experience and degree to which they discussed medication risks/benefits was not significantly associated with medication adherence (Sylvia et al.).

Health information. The third recommendation is appropriate delivery of health information. The National Standards for Diabetes Self-Management Education and Support (Funnell et al., 2010), stipulate that methods and materials used for diabetes teaching should be tailored to meet the needs of the patient. Multidimensional approaches that concurrently target mental health and general medical health have been found to be successful as shown in the Targeted Training in Illness Management study (Sajatovic et al., 2011). Priority areas for diabetes self-management education are diet and exercise, medication adherence, self-monitoring of blood glucose and psychosocial adjustment (Funnell et al.). Diabetes education improves clinical outcomes and quality of life.

Decisional control. Optimizing patients' self-management care is the fourth recommendation (McDevitt et al., 2003). An initial patient assessment should be completed to identify diabetes management skills needed and patients' abilities. A diabetes self-management plan is then developed and supported in collaboration with the mental health care team (McDevitt et al.). Basic diabetes competencies are introduced first as the tasks have a high likelihood of success then higher task demands are gradually introduced to maintain success. Individualized coaching and reinforcement are necessary to apply and maintain learning (Bachrach, 2000; McDevitt et al., 2003). Therapeutic 
relationships are important to reinforce self-management and encourage treatment adherence (Sylvia et al., 2013). Patients are encouraged to participate in their care and to make decisions about diabetes self-management, diet and exercise, medication adherence and self-monitoring of blood glucose (Reiss-Brennan et al., 2016). Decision making is determined by the patient's capability often indicated by the degree of independent living attained as well as medication and money management skills (McDevitt et al., 2003)

Professional-technical competency. The fifth recommendation is screening for diabetes. All patients should be screened for diabetes (McDevitt et al., 2003). Risk factors should be assessed including use of antipsychotic medications, family history of diabetes, overweight status, physical inactivity, ethnicity, history of gestational diabetes and high cholesterol. Patients at risk should be screened annually with the fasting or non-fasting plasma glucose testing. Screening should be done prior to starting antipsychotic medications (Haupt \& Newcomer, 2001) and thereafter every 2-3 months during the first year of antipsychotic use. Frequently prescribed second generation antipsychotic medications such as clozapine and olanzapine can cause impaired glucose tolerance, precipitate or accelerate onset of diabetes in susceptible patients and induce weight gain (Haupt \& Newcomer).

The sixth recommendation is treatment of pre-diabetes (McDevitt et al., 2003). Results indicating prediabetes are fasting blood glucose of $100-125 \mathrm{mg} / \mathrm{dl}$, A1C of 5.7\% $-6.4 \%$ and oral glucose tolerance test (OGTT) 2 hour blood glucose of $140 \mathrm{mg} / \mathrm{dl}-199$ $\mathrm{mg} / \mathrm{dl}$ (ADA, 2016). Prediabetes is associated with obesity especially visceral/ abdominal obesity, dyslipidemia with high triglycerides and/or low HDL cholesterol and hypertension (ADA, 2017). Lifestyle interventions associated with diet and exercise 
should be initiated first. If lifestyle interventions are ineffective after a 3 to 6-month trial, metformin should be considered (ADA). The American Diabetes Association (2017) recommended guidelines for metformin use suggest metformin should be considered in patients with prediabetes and additional risk factors such as age less than 60 years, BMI equal or greater $35 \mathrm{~kg} / \mathrm{m}^{2}$, prior gestational diabetes mellitus and raising hemoglobin A1C.

Provision of comprehensive diabetes care is the seventh recommendation (McDevitt et al., 2003). The National Standards for Diabetes Self-Management Education and Support and the American Diabetes Association guidelines support comprehensive care for diabetes patients (ADA, 2016; Funnell et al., 2010). Care should include a detailed history and physical exam, regular follow-ups, patient education and specialty referrals including for foot, dental and eye care. Services should be tailored to meet patient's needs based on cognitive deficits, self-management ability, environmental supports and status of the SPMI (McDevitt et al.).

Setting appropriate glycemic control goals is the eighth recommendation (McDevitt et al., 2003). Glycated hemoglobin (A1C) testing is recommended every 3-6 months (ADA, 2017). Glycated hemoglobin of 7 and below is associated with fewer microvascular complications. Glycemic control goals should be tailored to meet the needs and circumstances of the patient, with the goal of preventing complications. Diet, exercise and self-monitoring of blood glucose are important to maintain low A1C level (McDevitt et al.). 
Finally the ninth recommendation is provision of case management for outreach and care management services (McDevitt et al., 2003). Collaboration of medical and mental health care team ensures patients get the necessary immunizations, medications, health care supervision and support needed to be successful. Case managers assist the patients with work and/or treatment schedules, prioritization of events, and coordinate their appointments. Case management services play an important role in the success of the integrated service delivery model (Reiss-Brennan et al., 2016).

Next, the theoretical framework guiding this program evaluation will be presented. 


\section{Theoretical Framework}

The scope and practice of program evaluation has evolved and grown in complexity, as the importance and utility have become widely recognized (Saunders, Evans, \& Joshi, 2005). Evaluation is the systematic investigation of the merit (quality), significance, and the cost effectiveness (worth) of a program (CDC, 1999). The evaluation process monitors and documents program implementation, as well as facilitate understanding the relationship between specific elements of the program and program outcomes (Saunders et al.,2005). Evaluation has the essential role of both improving programs and satisfying accountability requirements (Levin \& Gregory, 2006). The CDC Framework for Program Evaluation (CDC, 1999) was utilized in this project to summarize and organize the crucial elements of program evaluation. The framework was designed to be applicable in any public health initiative and consists of two parts: steps of evaluation practice and standards of effective evaluation.

\section{Steps of Evaluation Practice}

The steps of evaluation practice facilitate a better understanding of the programs' context that is the history, setting, and organization (CDC, 1999). In addition, these steps improve how evaluations are perceived and conducted by tailoring evaluations to a particular public health effort. The framework consists of six interrelated steps that may be encountered in a nonlinear sequence. The steps, however, should be performed in a sequential manner, as earlier steps provide the foundation for subsequent steps/progress (CDC). 
The first step in the evaluation cycle is engaging the stakeholders, or partners. Stakeholders refer to persons or organizations that are involved in, or are affected by, the program outcomes (CDC, 1999). Primary users of the evaluation are also considered stakeholders. Partners are engaged in an inquiry to ensure their perspectives are understood, and elements important to them are included in the evaluation. The scope and level of stakeholder participation varies with the program. Engaging stakeholders increases the chances of evaluation outcomes being useful, avoids real or perceived conflicts of interest, and improves evaluation credibility (CDC). Stakeholders in this project potentially included clients, health care providers and administrators at the integrated care center. Given the vulnerability of the clients with SPMI, however, evaluation focused on engaging relevant health care providers and administrators.

Step two involves describing the program being evaluated to ensure its mission, objectives and strategies are clear to all (CDC, 1999). The program's stage of development, ability/capacity to influence change and its place in a larger context are described. The program-describing step sets the frame of reference for subsequent decision-making and the basis for comparisons with similar projects. Other aspects described are (a) statement of need/purpose, which describes the opportunities or problems to be addressed; (b) expected effects, referring to goals and objectives of the program; (c) activities to be performed and how they will lead to expected changes; and (d) resources, denoting all assets available for the program. Situations of mismatch between desired activities and available resources should be highlighted. The stage of program development, which may be planning, implementation or effects, is described within the context of program setting and environmental influences. A hypothesized 
sequence of events, or logic model, is displayed as a flow chart/map leading to the desired events (CDC, 1999). The student investigator explored the missions, goals and strategic plans of the two organizations that support the integrated care center.

The third step has a focus on evaluation design. Evaluation should be focused on issues of greatest concern to stakeholders, while effectively utilizing resources (CDC, 1999). Special attention is paid to the purpose of the evaluation and the users of the findings in order to develop feasible, useful and ethical evaluation strategies. Stakeholders' questions are used to develop methods and procedures that produce intended results. An agreement, which may be a legal contract or a detailed protocol, is put in place. The agreement summarize the procedures, clarifies roles, describes implementation of the evaluation plan, allocation of available resources and states safeguards in place to ensure that standards are met, especially those that protect human subjects (CDC). The student investigator presented the project's proposal to the Rhode Island College Institutional Review Board (IRB) and to the integrated care center management and was approved to carry on the project. Confidentiality paper work was also completed at the center.

The fourth step includes gathering credible evidence. Stakeholders should perceive information/evidence collected as authentic and relevant to answer their questions (CDC, 1999). Use of multiple mixed methods to gather, analyze and interpret data improves evidence credibility (CDC). The amount of evidence (quantity) and type of evidence (quality) are crucial to feasibility and accuracy of information. A balance between collecting enough data and assuring quality must be maintained. The use of both quantitative and qualitative data may help achieve that balance. Sources of data collection 
include interviews/surveys, documents review, and observations. Multiple indicators are not only necessary to track implementation and effects of a program, they increase accuracy of evaluation when used with multiple data sources (Levin \& Gregory, 2006). For the purposes and scope of this project, retrospective chart review method was used to collect quantitative data.

The fifth step is justifying conclusions. Evaluation conclusions are justified when they are consistent with the evidence gathered and favorably arbitrated against standards set by the stakeholders (CDC, 1999). Justifying conclusions involves analyzing data, interpreting the results, making judgments about the program and finally offering recommendations. Performance measures help to justify conclusions (CDC, 1999; Levin \& Gregory, 2006). The Clinical Practice Recommendations and Evidence-Based Guidelines for Integrated Care (McDevitt, et al., 2003) were used as standards against which components of the integrated care program were evaluated.

Lastly, the sixth step ensures the use and dissemination of lessons learned. Action-oriented recommendations should be formulated to ensure use of evaluation findings. Appropriate communications strategies utilizing the most effective formats and venues for different users should be used to disseminate the findings (Levin \& Gregory, 2006). The project's findings were compiled into the master's major paper and recommendations were made. The paper was given to the integrated care center and to Rhode Island College. 


\section{Standards of Effective Evaluation}

The second element of the CDC Program Evaluation Framework is a set of 30 standards used to assess the quality of evaluation activities and ensure a sound and fair evaluation process (CDC, 1999). The standards are organized into four groups: utility, feasibility, propriety and accuracy.

Utility standards ensure informational needs of users are satisfied (CDC, 1999). The standards address those who will be impacted by the evaluation, amount and type of information collected, values used in interpreting evaluation findings, and the clarity and timeliness of evaluation reports. Feasibility standards ensure the evaluation process is viable and practical (CDC). They emphasize use of practical, non-disruptive procedures, use of resources in a prudent fashion, and production of valuable findings. Propriety standards ensure the evaluation is ethical by developing protocols and agreements that guide the evaluation process (CDC). The standards protect the welfare of human subjects, weighs and discloses findings in a complete and balanced manner, and lastly addresses any conflicts of interest in an open and fair manner. Finally, accuracy standards ensure the evaluation process produces outcomes that are considered correct (CDC, 1999). The standards describe the program and its context, articulate the purpose and methods of the evaluation, employ systematic procedures to gather valid and reliable information, apply methods during analysis and synthesis, and produce impartial reports with justified conclusions (CDC).

Next, the methods will be presented. 


\section{Method}

\section{Purpose}

The purpose of this project was to evaluate components of a diabetes management program for persons with serious and persistent mental illness in an integrated primary and behavioral health care center in New England. Selected measures from the Clinical Practice Recommendations and Evidence-Based Guidelines for Integrated Care developed by McDevitt et al. (2003) were used to complete the evaluation

\section{Design}

The project utilized retrospective electronic chart reviews.

\section{Sample}

A simple random method was used to select the sample of 25 medical records from the target population of over 380 patient records. The inclusion criteria were: records of adult clients who received primary care at the health center; had a dual diagnosis of at least one serious mental illness and diabetes or $\mathrm{A} 1 \mathrm{C}>7 \%$; and received care at the health center for at least one year. Exclusions included records of adult clients who missed more than $25 \%$ of their scheduled appointments. The one year of care inclusion criteria allowed sufficient time to assess diabetes management, as clients with mental disorders may require more time to adjust to life style changes.

Site

The integrated health care center is a certified patient-centered medical home located in an urban setting where primary care, mental health and substance abuse treatment exist under one roof. Bilingual staff and shared medical and mental health records support efficient communications and effective care planning. The center 
includes medical and psychiatric treatment, smoking cessation programs and wellness programs that pair clients with health mentors who are certified personal trainers and experienced case managers to help achieve exercise and nutrition goals (The Providence Center, 2016).

\section{Measurement}

The project was guided by Clinical Practice Recommendations and Evidence-Based Guidelines for Integrated Care developed by McDevitt et al. (2003).The Center's diabetes guidelines/protocols and diabetic patient outcomes reports were reviewed for consistency with national standards and evaluation findings. The student investigator developed data collection tools including:

- Diabetes education content areas checklist: used to identify documented diabetes education content areas namely nutrition, exercises, medications, self-monitoring of blood sugar, foot care, dental care and smoking cessation. Smoking status, age and gender were also noted. The tool was adapted from the National Standards for Diabetes Self-Management Programs (Appendix A).

- The biophysical measures and missed appointment collection tool focused on the glycated hemoglobin (A1C), weight and the body mass index (Appendix B).

- A random number table.

\section{Procedures}

The program evaluation proposal approved by the administration of the integrated health care center and was reviewed by the Institutional Review Boards (IRB) at Rhode Island College. The manager of the integrated health care center provided paper lists of all patients with diabetes and one or more mental illness (387 patients). The lists 
contained patient names, medical record numbers, and name of primary care provider. To protect patients' personal health information, the paper lists were secured in a confidential cabinet folder in the manager's office and were accessed only while at the center. Only the randomly selected client charts were reviewed and no personal identifiers were recorded during data collection.

Due to the large size of the target population (over 380 [N] patient/charts), a simple random method (Appendix C) was used to select the sample of 25 patients (n). Numbers 1-25 was assigned to patients based on the random selection sequence. According to the health center reports, the proportions of males and females in the target population are almost the same and therefore sample stratification was not necessary. Medical record numbers were used to access retrospective electronic charts for selected patients only. Reviews were then conducted and data collected over a period of three months. The Center's diabetes guidelines/protocols and diabetic patient outcomes measures were reviewed for consistency with national standards and evaluation findings, hence consistency with the Clinical Practice Recommendations and Evidence-Based Guidelines for Integrated Care developed by McDevitt et al. (2003)

\section{Data Analysis}

The Microsoft Excel application was used to launch data into spreadsheets for better visualization and analysis. Excel was also used to calculate frequency distribution in terms of percentages, dispersion in terms of ranges and measures of central tendency; Mean and median

Next, study results will be presented. 


\section{Results}

\section{Center's Diabetes Guidelines/Protocols}

Review of diabetes guidelines/protocols at the center showed the standards of care outlined by the American Diabetes Association are followed. Diabetes neuropathy screening using the monofilament testing was recommended for all diabetes patients annually. No documentation was found on this screening. Protocol required reminder calls to be made on all prebooked appointments, medical chart previewed and documentation of pending and completed tasks. Completed and pending tasks including immunizations, screenings and tests were noted on every record reviewed. Physicians were to develop individualized care plans and be part of a care team whose goal was to improve diabetes control, obesity and hypertension by $5 \%$ over the baseline. Most patients were noted to have care plans and multidisciplinary care teams based on their needs. The center was committed to evidence based practice on chronic disease management and promoted self-management. Patient self-management goals included cutting down on smoking, 5\%weight loss in a quarter and $20 \%$ in a year and maintaining regular exercise. Most of the protocols reviewed needed to be updated.

\section{Record Reviews}

Of the 25 medical records reviewed, five (20\%) were excluded because the patients had missed more than $25 \%$ of scheduled appointments. This reduced the sample size to 20 . 
Table 1 illustrates diabetes education content areas checklist.

Table 1

Diabetes Education Content Areas Checklist

\begin{tabular}{|c|c|c|c|c|c|c|c|c|c|c|}
\hline \multicolumn{10}{|c|}{ Diabetes Education Content Areas Checklist } & \multirow[b]{2}{*}{$\begin{array}{l}\text { Smoking } \\
\text { Cessation }\end{array}$} \\
\hline No. & Gender & Age & \begin{tabular}{|l|} 
Smoking \\
Status
\end{tabular} & Exercise & Nutrition & Medication & \begin{tabular}{|l|} 
Self \\
Monitorin \\
$\mathrm{g}$ \\
of blood \\
glucose
\end{tabular} & $\begin{array}{l}\text { Foot } \\
\text { care }\end{array}$ & \begin{tabular}{|l} 
Dental \\
care
\end{tabular} & \\
\hline 1 & $\mathrm{~F}$ & 43 & yes & no & no & no & no & no & no & no \\
\hline 2 & $M$ & 60 & yes & no & yes & no & $\mathrm{n} / \mathrm{a}$ & no & no & no \\
\hline 3 & $\mathrm{~F}$ & 60 & yes & no & no & no & no & no & no & no \\
\hline 4 & $M$ & 46 & no & yes & yes & no & no & no & no & $\mathrm{n} / \mathrm{a}$ \\
\hline 6 & $\mathrm{~F}$ & 62 & no & yes & yes & no & no & yes & no & $\mathrm{n} / \mathrm{a}$ \\
\hline 7 & $\mathrm{~F}$ & 52 & yes & yes & Yes & yes & no & yes & no & no \\
\hline 8 & $\mathrm{~F}$ & 43 & yes & yes & yes & no & no & no & no & yes \\
\hline 9 & $\mathrm{~F}$ & 75 & yes & yes & yes & no & no & yes & no & yes \\
\hline 12 & $\mathrm{~F}$ & 46 & yes & yes & yes & no & no & yes & no & yes \\
\hline 13 & $\mathrm{~F}$ & 61 & no & yes & yes & no & no & yes & no & $\mathrm{n} / \mathrm{a}$ \\
\hline 14 & $\mathrm{~F}$ & 46 & yes & yes & yes & no & yes & Yes & yes & yes \\
\hline 15 & $M$ & 46 & yes & yes & yes & no & yes & yes & no & no \\
\hline 16 & $\mathrm{~F}$ & 50 & no & yes & yes & yes & no & no & no & $\mathrm{n} / \mathrm{a}$ \\
\hline 17 & $\mathrm{~F}$ & 53 & no & yes & yes & no & no & no & no & $\mathrm{n} / \mathrm{a}$ \\
\hline 19 & $\mathrm{~F}$ & 60 & no & yes & yes & no & no & no & no & $\mathrm{n} / \mathrm{a}$ \\
\hline 20 & $M$ & 38 & no & yes & yes & no & no & yes & no & $\mathrm{n} / \mathrm{a}$ \\
\hline 21 & $M$ & 51 & no & yes & yes & no & no & no & no & $\mathrm{n} / \mathrm{a}$ \\
\hline 23 & $\mathrm{~F}$ & 69 & no & yes & yes & no & no & no & no & $\mathrm{n} / \mathrm{a}$ \\
\hline 24 & $\mathrm{~F}$ & 61 & no & yes & yes & no & no & no & no & $\mathrm{n} / \mathrm{a}$ \\
\hline 25 & $\mathrm{~F}$ & 63 & yes & yes & yes & no & no & no & no & n/a \\
\hline
\end{tabular}

The median age of the patients was 52.5 years, with a range of 38-75 years. Most $(75 \%)$ of the participants were women. Fifty percent of all patients $(n=20)$ were smokers but only $40 \%(\mathrm{n}=10)$ of them received smoking cessation education. The majority of the 
patients $(n=20)$ received education on nutrition $(90 \%)$ and exercise $(85 \%)$ while only $10.5 \%(n=19)$ were educated on self-glucose monitoring and $10 \%(n=20)$ on medication. Merely $5 \%(n=20)$ of the patients were referred for dental care and $40 \%(n=20)$ for foot care.

Table 2 illustrates the biophysical measures and missed appointments.

Table 2

Biophysical Measures and Missed Appointments Data

\begin{tabular}{|c|r|r|r|r|r|r|r|r|r|r|r|}
\hline & \multicolumn{9}{|c|}{ Biophysical Measures and Missed Appointment Data } \\
\hline & \multicolumn{3}{|c|}{ AlC } & \multicolumn{3}{|c|}{ Weight } & \multicolumn{3}{|c|}{ BMI } & Appointments \\
\hline & & & & & & & & & & & \\
\hline \\
\hline
\end{tabular}


Three biophysical measurements indicated in Table 2 as 1,2 and 3 were obtained for AIC, weight in pounds (lbs.) and BMI. The number 1 reflected the most current measurement and 3 represented the baseline measure for the year December 2015 December 2016.

Based on the most current AIC, 65\% (n=20) of the patients had well controlled diabetes with AIC less than 7, ranging from 5.0-6.5.Ten percent $(n=20)$ of the patients had poorly controlled diabetes with AIC greater than 9; two clients had very high AIC of 10.2 and 10.9. Fifty percent $(n=20)$ of the patients had minor (less than 1$)$ increase of AIC from baseline though significant increases of 1.4 and 4.2 were also noted. Thirty five percent $(n=20)$ improved their AIC mostly by 1 or less points, while some improved significantly by $3.3 \%$ and $5.8 \%$. One person maintained baseline level. The current AIC median and average were 6.25 and 6.78 respectively as opposed to 6.5 and 6.95 at baseline.

The average current weight was $202.75 \mathrm{lb}$. with ranges between $141-259 \mathrm{lbs}$. The average baseline weight was 196.5 lbs. With ranges between 141 and 257.lbs. Thirty percent $(n=20)$ of the participants lost weight losing between 2-6 lbs. Most participants $(65 \%)$ however gained weight ranging from 1-38.25 lbs. Two patients had significant gains of 34 and $38.25 \mathrm{lbs}$. The baseline BMI median was 34.3 with a range of 24.3-47.4, and the current BMI median was 35.35 with range of 23.9-48.8.

Sixty percent $(\mathrm{n}=20)$ of all participants did not miss any scheduled appointments, $66.7 \%(n=12)$ of them gained weight and increased AIC from baseline. Among them 
were those who gained more than 33 lbs. Fifty eight percent $(n=12)$ had well controlled AIC below 7 while $25 \%(n=12)$ had poorly controlled diabetes with AIC greater than 8 .

Next the summary and conclusion will be presented. 


\section{Summary and Conclusions}

The Clinical Practice Recommendations and Evidence-Based Guidelines for Integrated Care developed by McDevitt et al. (2003) were used to evaluate a diabetes management program for persons with serious and persistent mental illness, in an integrated primary and behavioral health care center in New England. The project utilized retrospective electronic chart review design to gather data. A simple random method was used to select a sample population of 25 patient medical records from the target population of over 380 patient records. The inclusion criteria were: records of adult clients who received primary care at the health center, had a dual diagnosis of at least one serious mental illness and diabetes or AIC greater than 7 and received care at the health center for at least one year. Exclusions included records of adult clients who missed more than $25 \%$ of their scheduled appointments.

The center's diabetes guidelines/protocols were reviewed and found to be consistent with national standards and evaluation findings. The student investigator developed two data collection tools namely the diabetes education content areas checklist and the biophysical measures and missed appointment collection tool. The diabetes education content areas were nutrition, exercises, medications, self-monitoring of blood sugar, foot care, dental care and smoking cessation. Smoking status of patients, age and gender were also noted. The tool was adapted from the National Standards for Diabetes Self-Management Programs The biophysical measures and missed appointment collection tool focused on the glycated hemoglobin, weight and the body mass index. Percentage of missed appointments was calculated. 
The program evaluation proposal was submitted to and approved by the Institutional review Boards at Rhode Island College and the integrated health care center in December 2016. Paper lists of all patients with diabetes and one or more mental illness were provided by the center's manager. To protect patients' personal information, the paper lists were secured in a confidential cabinet folder in the manager's office and were accessed only while at the center. Medical record numbers were used to access retrospective electronic charts for selected patients only. Reviews were then conducted and data collected over a period of three months: January 2017 through March 2017.

Missing appointments presented as a big problem for the center as evidenced by exclusion of $20 \%(n=25)$ of patients who missed more than $25 \%$ of their scheduled appointments. Of the remaining patients $25 \%(\mathrm{n}=20)$ missed $25 \%$ of their appointments. This may be attributed to lack of transportation, moving to different locations, or simply forgetting. To address the problem, patients are called and reminded of their appointments a day earlier. Those who miss appointments receive a call the same day to reschedule if they are not available a post card reminder is sent to their residence. Integrated care attempts to address this issue by providing all services under one roof Missing appointments was not directly addressed by the Clinical Practice Recommendations and Evidence-Based Guidelines.

Unexpected finding noted was those who kept all appointments did not have better results than those who missed. Sixty percent of all patients $(n=20)$ did not miss any appointment, of these clients $67 \%(n=12)$ gained weight, $58 \%(n=12)$ had AIC $<7$ and $25 \%(\mathrm{n}=12)$ had AIC $>8$. The center focused on exercise and nutrition education areas as shown by the high documented percentages $85 \%(n=20)$ and $90 \%(n=20)$ respectively. 
Other important education content areas such as self-glucose monitoring and medications were documented as offered to $20 \%(n=20)$ and $5 \%(n=20)$ of patients respectively. Most patients were on one or combinations of diabetes oral medication namely Metformin, Glyburide, Glipizide, Januvia, Glimepiride and long acting insulin. They also checked their blood glucose. Metformin was recommended by the guidelines. Fifty percent of the patients $(n=20)$ smoked, yet only $40 \%(n=10)$ were documented having received smoking cessation education. Smokers have higher risk for diabetes complications including cardiovascular, renal, retinopathy, neuropathy and poor circulation /amputations (CDC, 2014). Only one patient (5\%) was referred to a dentist and $38 \%(n=20)$ were referred for foot care. Referrals and exams are important part of preventative care to delay or avoid diabetes complication. There may be a possibility that education was offered but not documented.

Most $65 \%(n=20)$ of participants gained weight as opposed to $30 \%(n=20)$ who lost weight. The average weight was $202.8 \mathrm{lbs}$. with an average weight gain of $6.75 \mathrm{lbs}$. This may be attributed to various factors such as the high cost of health foods namely fruits and vegetables and accessibility to cheap high salt, high sugar and carbohydrates foods. Many patients reported irregular active physical activities. Walking may be the activity of choice for majority of clients given the cost of gymnasium and scares resources. Like with other outdoor activities, walking is dependent on the weather Most of these patients were also on antipsychotic medications which may cause weight gain. The median BMI was 35 which indicate obesity. Interestingly however the A1C of $65 \%$ $(n=20)$ of the patients were very well controlled with A1C below 7, 25\% (n=20) had A1C range of 7.3-8.2 and only $10 \%(n=20)$ had poorly controlled diabetes at AIC greater than 
9. The positive results may be attributed to personalized medication regimen and compliance to treatment.

Two methodological limitations were noted namely a small sample size and missing data. A small sample size of 20 client charts was used for the study. This may have resulted to difficulty in finding significant relationships from the data. The sample size was comprised of mostly $(75 \%)$ women. A larger, gender balanced sample should be used in future studies to ensure a more representative population and generalization of findings. Missing data was encountered while collecting biophysical information. Some charts had only two values of a particular biophysical measure while others charts had several values of the same measure. The last 3 values of AIC, weight and BMI were collected and labeled 1, 2, and 3. One was most current measurement and 3 represented the baseline measure. Where only two values were available, the most current value was labeled 1 and the other became the base line. An investigator limitation experienced was access to the electronic health records. The investigator's computer access was terminated amidst data collection for expired access period. It took two weeks to regain computer access which regressed data collection efforts.

Diabetes education remains a key element in the management of diabetes. Education should be tailored to meet the specific needs of the patient and cover relevant content areas. Collaboration of medical and mental health care team is important to ensure the wellbeing of this vulnerable population. The role of the care manager is essential in the success of the integrated service delivery model. Care managers assist the patients with work and/or treatment schedules, prioritization of events, coordinate their appointments and check on the patient to make sure they are doing alright. The findings 
of this project underscore the complexity and confounding nature of factors influencing the disease process in this population

Next recommendations and implications for advanced nursing practice will be presented. 


\section{Recommendations and Implications for Advanced Nursing Practice}

There is a complex, multifactorial association between diabetes and psychiatric disorders that compounds the treatment and management of the diseases. Determinants of health work against this vulnerable population leaving them exposed to natural courses and consequences of poverty among them limited or no access to health care. It is therefore imperative that advanced practice public health nurses (APHN) invest in continued education and technology training to ensure efficient assessment and analysis of population health data and authentic determination of population needs and opportunities.

Implication for practice calls for the APHNs to work with communities and populations as equal partners focusing on primary prevention and health promotion. Advanced practice public health nurses should collaborate with other community partners to conduct community assessments, identify population's needs and opportunities, create and implement programs as well as identify expected outcomes in the health status of the population. Projects conducted should encourage community participation across socialeconomic status and focus on community assets and resources rather than on deficits. Effectiveness of the programs should be constantly evaluated to ensure success and lasting improvements in the population. Advanced practice public health nurses msut be aware of existing community resources and refer people to the appropriate services based on their needs.

It is important for the APHNs to join professional organizations and participate in efforts to lobby for policies and laws that favor vulnerable populations. They should 
promote and support the development of programs, policies, and services that provide interventions to improve the health status of this populations. Vulnerable populations are often marginalized and APHNs should stand up and advocate for those who have no voice. With improved technology and advancement in science and medicine, further research is necessary to identify more effective techniques to manage mental health comorbidities and promote population health status. 


\section{References}

Agency for Healthcare Research and Quality (2013).Benefits of case management for chronic illness. Retrieved from http://www.ahrq.gov/news/newsletters/researchactivities/13feb/0213ra15.html

Amador, X. F., Flaum, M., Andreasen, N. C., Strauss, D. H., Yale, S. A., Clark, S. C., \& Gorman, J. M. (1994).Awareness of illness in schizophrenia and schizoaffective and mood disorders. Archives of General Psychiatry, 51, 826-836.Retrieved from http://www.ncbi.nlm.nih.gov/pubmed/7944872

American Diabetes Association (2016). American Diabetes Association Standards of Medical Care in Diabetes Abridged for primary care providers. Clinical Diabetes, 34(1). Retrieved from http://clinical.diabetesjournals.org/content/34/1/3

American Diabetes Association, (2017).American Diabetes Association Standards of Medical Care in Diabetes. Care Diabetes Journals 40(1). Retrieved from http://professional.diabetes.org/sites/professional.diabetes.org/files/media/dc_40_ s1_final.pdf

American Diabetes Association, (2016).Diagnosing diabetes and learning about prediabetes. Retrieved from http://www.diabetes.org/diabetesbasics/diagnosis/?referrer=https://www.google.com/

Anderson, R. M., Funnell, M. M., Fitzgerald, J. T., \& Marrero, D. G. (2000). The diabetes empowerment scale: A measure of psychosocial self-efficacy. Diabetes Care, 23, 739-743. Retrieved from https://www.researchgate.net/profile/Martha_Funnell/publication/12477287_The_ 
Diabetes_Empowerment_Scale_a_measure_of_psychosocial_selfefficacy/links/0046351caf8ddec968000000.pdf

Bachrach, L. L. (2000). Psychosocial rehabilitation and psychiatry in the treatment of schizophrenia-what are the boundaries? [Review.] Acta Psychiatrica Scandinavica, 102 (Suppl.), 6-10. Retrieved from http://www.ncbi.nlm.nih.gov/pubmed/11261643

Balhara, Y. P. S. (2011). Diabetes and psychiatric disorders. Indian Journal of Endocrinology and Metabolism,15(4), 274-283.Retrieved from http://www.ncbi.nlm.nih.gov/pmc/articles/PMC3193776/

Bandura, A. (1977). Self-efficacy: Toward a unifying theory of behavioral change. Psychological Review, 84(2), 191-215. Retrieved from https://www.uky.edu/ eushe2/Bandura/Bandura1977PR.pdf

Centers for Disease Control and Prevention (1999). Framework for program evaluation in public health. Morbidity and Mortality Weekly Report, 48(11), 1-58. Retrieved from ftp://ftp.cdc.gov/pub/Publications/mmwr/rr/rr4811.pdf

Centers for Disease Control and Prevention (2011). Mental illness surveillance. Retrieved from http://www.cdc.gov/mentalhealthsurveillance/fact_sheet.html

Centers for Disease Control and Prevention, National Center for Chronic Disease Prevention and Health Promotion, Office on Smoking and Health, (2014). The health consequences of smoking-50 Years of progress: A report of the Surgeon General. Retrieved from https://www.surgeongeneral.gov/library/reports/50-yearsof-progress/exec-summary.pdf 
Centorrino F., Mark T. L., Talamo A., Oh K., \& Chang J. (2009). Health and economic burden of metabolic comorbidity among individuals with bipolar disorder. Journal of Clinical Psychopharmacol, 29(6), 595-600.Retrieved from http://www.ncbi.nlm.nih.gov/pubmed/19910727/

Chew, B.-H., Shariff-Ghazali, S., \& Fernandez, A. (2014). Psychological aspects of diabetes care: Effecting behavioral change in patients. World Journal of Diabetes, 5(6), 796-808.Retreived from https://www.ncbi.nlm.nih.gov/pmc/articles/PMC4265866/

Colton, C. W., \& Manderscheid, R. W. (2006).Congruencies in increased mortality rates, years of potential life lost, and causes of death among public mental health clients in eight states. Preventing Chronic Disease, 3(2), A42.

De Hert, M., Correll, C. U., Bobes, J., Cetkovich-Bakmas, M., Cohen, D., Asai, I., Leucht, S. (2011). Physical illness in patients with severe mental disorders: Prevalence, impact of medications and disparities in health care. World Psychiatry, 10(1), 52-77. Retrieved from http://www.ncbi.nlm.nih.gov/pmc/articles/PMC3048500/

Funnell, M. M., Brown, T. L., Childs, B. P., Haas, L. B., Hosey, G. M., Jensen, B., Weiss, M. A. (2010). National Standards for Diabetes Self-Management Education. Diabetes Care, 33 (Suppl 1), S89-S96. Retrieved from http://www.ncbi.nlm.nih.gov/pmc/articles/PMC2797385/

Haas, L., Maryniuk, M., Beck, J., Cox, C. E., Duker, P., Edwards, L., Youssef, G., on behalf of the 2012 Standards Revision Task Force. (2012). National Standards for Diabetes Self-Management Education and Support. Diabetes Care, 35(11), 23932401. Retrieved from http://www.ncbi.nlm.nih.gov/pmc/articles/PMC3476915/ 
Haupt, D., W., and Newcomer, J., W., (2001). Hyperglycemia and antipsychotic medications Clinical Psychiatry 62(27): 15-26. Retrieved from https://www.ncbi.nlm.nih.gov/pubmed/11806485

Hemingway, S., Trotter, F., Stephenson, J., \& Holdich, P. (2013). Diabetes: increasing the knowledge base of mental health nurses. British Journal of Nursing, 22(17), 991-995.Retrieved from http://0web.a.ebscohost.com.helin.uri.edu/ehost/pdfviewer/pdfviewer?vid=15\&si d=e6a7b149-a260-4e29-ac65-a0f2064668ad\%40sessionmgr4001\&hid=4107

Huppert, J. D., Weiss, K. A., Lim, R., Pratt, S., \& Smith, T. E. (2001). Quality of life in schizophrenia: Contributions of anxiety and depression. Schizophrenia Research, 51, 171-180. Retrieved from http://www.ncbi.nlm.nih.gov/pubmed/11518637

International Diabetes Federation (2006). Global guideline for type 2 diabetes: Recommendations for standard, comprehensive, and minimal care. Diabetic Medicine, 23(6), 579-593.Retrieved from http://web.a.ebscohost.com/ehost/pdfviewer/pdfviewer?sid=2b6b8f81-a023-48f9990a-95572e2ed3e2\%40sessionmgr4002\&vid=9\&hid=4101

Kopelowicz, A., Liberman, R. P., Zarate, R., ( 2006). Recent advances in social skills training for schizophrenia. Schizophr Bull 2006; 32 suppl_1. Retreived for https://academic.oup.com/schizophreniabulletin/article/32/suppl_1/S12/1915328/Rec ent-Advances-in-Social-Skills-Training-for

Levin, M. S., \& Gregory, W. (2006). A six-step model for evaluation of communitybased physical activity programs. Preventing Chronic Disease, 3(1), A24. Retrieved from http://www.ncbi.nlm.nih.gov/pmc/articles/PMC1500959/ 
Macdonald, E. M., Pica, S., Macdonald, S., Hayes, R. L., \& Baglioni, A. J., Jr. (1998). Stress and coping in early psychosis: Role of symptoms, self-efficacy, and social support in coping with stress. British Journal of Psychiatry (Suppl.), 172, 122127. Retrieved from http://www.ncbi.nlm.nih.gov/pubmed/9764138

McDevitt, J., Snyder, M., Breitmayer, B., Paun, O., \& Wojciechowski, E. (2003). Diabetes management in the context of serious and persistent mental illness. Clinical practice recommendations evidence-based guidelines for integrated care. Retrieved from file://C:/Documents\%20and\%20Settings/user/My\%20Documents/Downloads/Cli nical_Practice_Recommendations\%20(2).pdf

Nicolucci, A., Burns, K., Holt, R., Comaschi, M., Hermanns, N., Ishii, H. \&, Kokoszka et al., (2013). Diabetes attitudes, wishes and needs second study (DAWN2): Crossnational benchmarking of diabetes-related psychosocial outcomes for people with diabetes. Diabetic Medicine, 30(7), 767-777. Retrieved from http://web.a.ebscohost.com.ric.idm.oclc.org/ehost/pdfviewer/pdfviewer?sid=8006 ab88-dfbd-4aa3-993d-3751e56366fe\%40sessionmgr4006\&vid=7\&hid=4204

Olfson M, Gerhard T, Huang C, Crystal S, Stroup T.S. (2015). Premature mortality among adults with schizophrenia in the United States. JAMA Psychiatry 72(12):1172-1181. Retrieved from https://www.ncbi.nlm.nih.gov/pubmed/26509694

Reiss-Brennan, B., Brunisholz, K., Dredge, c., Pascal, B., Grazier, K., Wilcox, A., Savitz, L., James, B. (2016).Association of integrated team-based care with health care quality, utilization, and cost. Journal of the American medical Association, 316 
(88) Retrieved from

http://jamanetwork.com/pdfaccess.ashx?url=/data/journals/jama/935665

Rethink Mental Illness (2009). Health inequalities are killing people with mental illness. Retrieved from http://www.politics.co.uk/opinion-formers/rethink/article/rethinkhealth-inequalities-are-killing-people-with-mental-i

Sajatovic, M., Dawson, N. V., Perzynski, A. T., Blixen, C. E., Bialko, C. S., McKibbin, C. L., Fuentes-Casiano, E. (2011). Optimizing care for people with serious mental illness and comorbid diabetes. Psychiatric Services (Washington, D.C.), 62(9), 1001-1003. Retrieved from https://www.ncbi.nlm.nih.gov/pmc/articles/PMC4497574/

Saunders, R. P., Evans, M. H., \& Joshi, P., (2005). Developing a process evaluation plan for assessing health promotion program implementation: A how-to guide. Health Promotion Practice, 6(2), 134-147. Retrieved from http://bvs.panalimentos.org/local/file/INCLUSIONES2008/7GSS_PULSENET_A VAN\%C7ADO2008/II\%20Curso\%20Avanz\%20WGSS-Taller\%20WGSSPulseNet/11_Documentos/Literature\%20Review/Course\%20Impact\%20Evaluatio n/Designing\%20process\%20evaluations.pdf

Substance Abuse and Mental Health Services Administration. Center for Behavioral Health Statistics and Quality, (2015). National survey on drug use and health. Retrieved from https://www.samhsa.gov/data/sites/default/files/NSDUH-FFR12015/NSDUH-FFR1-2015/NSDUH-FFR1-2015.htm

Substance Abuse and Mental Health Services Administration (2014). Projections of national expenditures for treatment of mental and substance use disorders, 2010- 
2020.Retrieved from http://store.samhsa.gov/shin/content/SMA14-4883/SMA144883.pdf

Sylvia, G., Hay, A., Ostacher, M. J., Miklowitz, D. J., Nierenberg, A. A., Thase, M. E...,Perlis, R. H.,. (2013). Association between therapeutic alliance, care satisfaction, and pharmacological adherence in bipolar disorder. Journal of Clinical Psychopharmacology, 33(3). Retrieved from https://www.ncbi.nlm.nih.gov/pmc/articles/PMC3873324/

The Providence Center, (2016). Everything you need to be health under one roof. Retrieved from https://providencecenter.org/services/integrated-primary-andbehavioral-care/pchc-at-north-main

Walker, R. J., Smalls, B. L., Hernandez-Tejada, M. A., Campbell, J. A., Davis, K. S., \& Egede, L. E. (2012). Effect of diabetes fatalism on medication adherence and Self-Care Behaviors in Adults with Diabetes. General Hospital Psychiatry, 34(6), 598-603.Retrieved from https://www.ncbi.nlm.nih.gov/pubmed/21636163

Ward, M., \& Druss, b. (2015). The epidemiology of diabetes in psychotic disorders. The Lancet Psychiatry, 2 (5) 431- 451. Retrieved from http://www.thelancet.com/journals/lanpsy/article/PIIS2215-0366 (15)000073/abstract 


\section{Appendix A}

Diabetes Education Content Areas Checklist

Diabetes Education Content Areas Checklist for December 2015-December 2016

\begin{tabular}{|c|c|c|c|c|c|c|c|c|c|c|}
\hline $\begin{array}{l}\text { Patient } \\
\text { number }\end{array}$ & Gender & Age & $\begin{array}{l}\text { Smoking } \\
\text { Status }\end{array}$ & Exercise & Nutrition & Medication & $\begin{array}{l}\text { Self } \\
\text { Monitoring } \\
\text { of blood } \\
\text { glucose }\end{array}$ & $\begin{array}{l}\text { Foot } \\
\text { care }\end{array}$ & $\begin{array}{l}\text { Dental } \\
\text { care }\end{array}$ & $\begin{array}{l}\text { Smoking } \\
\text { Cessation }\end{array}$ \\
\hline & & & & & & & & & & \\
\hline & & & & & & & & & & \\
\hline & & & & & & & & & & \\
\hline & & & & & & & & & & \\
\hline & & & & & & & & & & \\
\hline & & & & & & & & & & \\
\hline & & & & & & & & & & \\
\hline & & & & & & & & & & \\
\hline & & & & & & & & & & \\
\hline & & & & & & & & & & \\
\hline & & & & & & & & & & \\
\hline & & & & & & & & & & \\
\hline & & & & & & & & & & \\
\hline & & & & & & & & & & \\
\hline & & & & & & & & & & \\
\hline & & & & & & & & & & \\
\hline & & & & & & & & & & \\
\hline & & & & & & & & & & \\
\hline
\end{tabular}


Appendix B

Biophysical Measures and Missed Appointment Collection Tool

Biophysical Measures and Missed Appointment For December 2015-December 2016

\begin{tabular}{|c|c|c|c|c|c|c|c|c|c|c|c|}
\hline & \multicolumn{3}{|c|}{ AIC } & \multicolumn{3}{|c|}{ Weight } & \multicolumn{3}{|c|}{ BMI } & \multicolumn{2}{|c|}{ Appointments } \\
\hline $\begin{array}{l}\text { Patient } \\
\text { number }\end{array}$ & 1 & 2 & 3 & 1 & 2 & 3 & 1 & 2 & 3 & Appts & $\begin{array}{l}\% \text { Appt } \\
\text { Missed }\end{array}$ \\
\hline & & & & & & & & & & & \\
\hline & & & & & & & & & & & \\
\hline & & & & & & & & & & & \\
\hline & & & & & & & & & & & \\
\hline & & & & & & & & & & & \\
\hline & & & & & & & & & & & \\
\hline & & & & & & & & & & & \\
\hline & & & & & & & & & & & \\
\hline & & & & & & & & & & & \\
\hline & & & & & & & & & & & \\
\hline & & & & & & & & & & & \\
\hline & & & & & & & & & & & \\
\hline & & & & & & & & & & & \\
\hline & & & & & & & & & & & \\
\hline & & & & & & & & & & & \\
\hline & & & & & & & & & & & \\
\hline & & & & & & & & & & & \\
\hline
\end{tabular}

